\title{
IMPACTO DA COVID-19 NA UTILIZAÇÃO DE SERVIÇOS PARA VACINAS DO PROGRAMA NACIONAL DE IMUNIZAÇÕES
}

\author{
Érica Marvila Garcia' \\ ORCID: 0000-0001-9477-2383
}

Clebson Verissimo da Costa Pereira"

ORCID: 0000-0003-4408-2645

Ana Paula Sayuri Sato' ORCID: 0000-0001-8601-5884

'Faculdade de Saúde Pública, Universidade de São Paulo. São Paulo, SP.

"Sociedade Brasileira de Imunizações na Paraíba. João Pessoa, PB.

Autora Correspondente:

Érica Marvila Garcia

E-mail: ericamarvila@usp.br

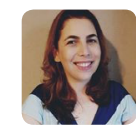

Como citar:

Garcia EM, Pereira CVC, Sato APS. Impacto da COVID-19 na utilização de serviços para vacinas do Programa Nacional de Imunizações. In: Silva TMR, Lima MG, (Orgs.). Estratégias de vacinação contra a COVID-19 no Brasil: capacitação de profissionais e discentes de enfermagem. Brasilia, DF: Editora ABen; 2021. P 14-20. (Série enfermagem e pandemias, 6). https://doi.org/10.51234/aben.21.e08.c02

Revisor: Renato Kfouri. Médico Pediatra. Primeiro secretário da SBIm e presidente do Departamento Científico de Imunizações da Sociedade Brasileira de Pediatria (SBP).

\section{INTRODUÇÃO}

Criado em 1973, o Programa Nacional de Imunizações (PNI) brasileiro resultou das estratégias de vacinação bem-sucedidas para a erradicação da varíola e da crescente preocupação com a disponibilidade dos imunobiológicos no estabelecimento de um sistema nacional de saúde. Alicerçado nos princípios doutrinários do Sistema Único de Saúde (SUS), o PNI tem como objetivo controlar, eliminar e erradicar as doenças imunopreveníveis. Atualmente, o PNI oferece, gratuitamente, 15 tipos diferentes de vacinas para crianças, nove para adolescentes e cinco para adultos e idosos, contando com mais de 38 mil salas de vacinas, nos 5.570 municípios brasileiros, por meio de uma rede articulada, hierarquizada e integrada (1).

Ao longo de toda a história, vários desafios foram enfrentados pelo PNI. Na década de 1980, havia piores coberturas vacinais em segmentos mais pobres, em 2007, a cobertura era mais baixa entre os segmentos mais ricos e nos extremos pobres da população e, a partir de 2016, houve o declínio de 10 a 20 pontos percentuais das coberturas vacinais em geral ${ }^{(2-5)}$. Algumas possíveis explicações foram levantadas para esta queda: a) a diminuição da percepção de risco das doenças; b) o aumento da percepção de risco de eventos adversos pós-vacina; c) a crise político-econômica, com a redução do apoio governamental e a presença de subfinanciamento ao SUS; d) a disseminação de informações distorcidas sobre vacinas por meio das redes sociais; e) o acesso limitado às salas de vacina pelo seu restrito horário de funcionamento; f) a capacitação inadequada do profissional da saúde lotado na sala de vacinação e a sua alta rotatividade e; $g$ ) o aumento da hesitação vacinal. Essa última, tem ganhado importância em várias partes do globo e se caracteriza pelo atraso em aceitar ou pela recusa em receber a vacina, independentemente de sua disponibilidade e do acesso aos serviços de saúde ${ }^{(5-8)}$. 
O mais recente desafio foi configurado pelo atual cenário pandêmico da COVID-19, que impôs uma sobrecarga aos profissionais e serviços de saúde. O temor populacional ao vírus e o distanciamento social instituído têm acarretado a precarização dos cuidados de saúde de rotinas preventivas e do acesso aos serviços. A priorização da atenção à urgência/emergência e dos cuidados intensivos prejudicou e impactou os cuidados da atenção básica, dentre eles, a vacinação ${ }^{(9-10)}$. A fragilização dos programas de imunização ocorrida nesse período é fenômeno mundial, e configura-se um acréscimo aos danos causados pela hesitação vacinal, ampliando os desafios na busca por coberturas vacinais elevadas e homogêneas para o controle das doenças imunopreveníveis. Assim, diante do contexto atual, em que as vacinas contra o coronavírus tornam-se essenciais para a vida e para a mitigação da pandemia, novos desafios se colocam ao PNI.

\section{O IMPACTO GLOBAL DA PANDEMIA DE COVID-19 NA UTILIZAÇÃO DE SERVIÇOS DE SAÚDE PARA A VACINAÇÃO}

Doenças emergentes e reemergentes constituem um incessante risco à humanidade. Nas últimas décadas, várias epidemias de doenças infecciosas, com grandes ameaças a sociedade foram vivenciadas em todo o mundo ${ }^{(11)}$, tais como: síndrome respiratória aguda grave, na China, em 2002; pandemia da gripe H1N1, no México, em 2009; síndrome respiratória do Oriente Médio, na Arábia Saudita, em 2012; surto do vírus Ebola, na África Ocidental, no final de 2013; surto do vírus Zika, no Brasil, em 2015; surto pelo vírus Lassa, na Nigéria, em 2018; e, por fim, a pandemia pelo SARS-CoV-2, em curso (COVID-19) ${ }^{(12)}$.

Relatado pela primeira vez em dezembro de 2019, na cidade de Wuhan (China), o SARS-CoV-2 se espalhou rapidamente pelo mundo, desencadeando uma pandemia em apenas três meses. Essa disseminação acelerada desencadeou uma busca intensa por ações para contê-lo, e, na ausência de tratamentos ou vacinas eficazes, foram implementadas as intervenções não farmacológicas, como lockdown, cancelamento de atividades, distanciamento social obrigatório, uso contínuo de máscaras, fechamento de escolas e restrições de viagens ${ }^{(13-14)}$.

A pandemia da COVID-19 acarretou uma grave crise nos diversos setores da sociedade, em especial na saúde; seu impacto variou entre os países, assim como a escolha das medidas de controle. Entretanto, se observou, em diferentes localidades, a redução do uso dos serviços de saúde ${ }^{(15)}$. Durante os períodos de quarentena, os serviços de saúde de rotina e procedimentos cirúrgicos eletivos foram interrompidos em muitos estabelecimentos e os profissionais de saúde foram reorganizados de tal forma a apoiar ou priorizar o atendimento de um número crescente de pacientes com COVID-19 ${ }^{(16)}$.

O atual cenário pandêmico expôs as fragilidades dos sistemas de saúde ao redor do mundo, o colapso em muitos destes, oriundo da alta demanda por atendimento de alta complexidade, atingiu até mesmo países de alta renda e com sistemas públicos de saúde bem estruturados ${ }^{(17)}$. Essa situação se agravou ainda mais quando países de baixa e média renda, com sistemas já sob pressão, sofreram, ainda que temporárias, interrupções de seus programas de saúde, abrindo a porta para o ressurgimento de outras doenças ${ }^{(18)}$.

Afetados diretamente por esse cenário, os programas de imunizações observaram a redução das coberturas vacinais e o possível reaparecimento de doenças imunopreveníveis. O receio dos pais em relação a transmissão do vírus, as restrições de movimento, as políticas de bloqueio, as mudanças de prioridades para COVID-19 e a escassez de vacinas por questões de logística de entrega contribuíram para a interrupção, adiamento, reorganização ou suspensão total dos serviços de imunização, em todas as faixas etárias ${ }^{(16,19-20)}$, além da suspensão de algumas ações de imunização em massa ${ }^{(21-22)}$.

Nesse período, a Organização Mundial de Saúde (OMS) e a Organização Pan-Americana da Saúde (OPAS), considerando três cenários de disponibilidades de serviços de saúde, publicaram recomendações acerca das ações de vacinação, dentre elas a suspensão temporária das campanhas de imunização em massa e a manutenção da vacinação de rotina, este último, em locais onde houvesse capacidade operacional de recursos humanos e fornecimento de vacinas preservados, respeitando-se as medidas de controle da pandemia. Assim, 
mais da metade dos 129 países interromperam de forma moderada, grave ou totalmente seus serviços de vacinação no ano de 2020, outros, incluindo o Brasil, adiaram as campanhas de imunização nos primeiros cinco meses da pandemia ${ }^{(23-24)}$.

Essa suspensão impactou diretamente as coberturas vacinais, colocando em risco pelo menos 80 milhões de crianças menores de um ano ${ }^{(16,23)}$. Na França, as vacinas infantis diminuíram em quase um quarto ${ }^{(25)}$. A Associação Espanhola de Pediatria relatou uma queda de $5 \%$ a $60 \%$ na cobertura vacinal ${ }^{(26)}$. Na Inglaterra, um declínio nas doses administradas foi observado para a vacina de sarampo, caxumba e rubéola (3,7\%) e vacina hexavalente $(3,5 \%)^{(27)}$. Nos EUA, foi observado um declínio de aproximadamente $75 \%$ nas vacinas solicitadas por médicos, além de um declínio de $21,5 \%$ nas administrações de vacinas de sarampo ${ }^{(28)}$. Em Serra Leoa, foi relatado um declínio de $57 \%$ no número de crianças que visitam a clínica para vacinação(29), no Paquistão, a cobertura caiu em mais de $50 \%$ para todas as vacinas ${ }^{(30)}$ e no Líbano, as taxas de aplicação de todas as vacinas caíram aproximadamente $50 \%{ }^{(31)}$.

No Brasil, a pandemia foi um desafio adicional aos obstáculos já enfrentados com as recentes quedas nas coberturas vacinais e a epidemia de sarampo, que atingiu vários estados ${ }^{(32-33)}$. De acordo com os dados do Sistema de Informação do Programa de Nacional de Imunização (SI-PNI), em 2020, com exceção da vacina pentavalente, o país exibiu uma considerável redução nos números de doses de vacinas aplicadas. Em comparação com 2019, houve diminuição na cobertura vacinal de: i) $15,8 \%$ da vacina de hepatite $B$ até 30 dias de vida (cobertura em 2020 de 62,8\%); ii) 13,6\% da $1^{\text {a }}$ dose da vacina Tríplice Viral (cobertura em 2020 de 79,5\%); iii) 13,4\% da vacina BCG (cobertura em 2020 de 73,3\%); iv) 10,1\% da vacina contra hepatite A (cobertura em 2020 de 74,9\%); v) 9,2\% da vacina meningocócica C (cobertura em 2020 de 78,2\%); vi) 8,4\% da vacina rotavírus humano (cobertura em 2020 de 77\%); vii) 8,3\% da vacina poliomielite (cobertura em 2020 de 75,9\%) e; vii) 8,1\% da vacina pneumocócica (cobertura em 2020 de 81,0\%). Assim, somente a cobertura da vacina pneumocócica esteve acima dos $80 \%$. Ainda, mais de $50 \%$ dos municípios ficaram abaixo das metas de coberturas estabelecidas pelo PNI para as vacinas BCG e rotavírus humano que são de $90 \%$ e para as demais que são de $95 \%{ }^{(34)}$.

Quando os programas de imunizações são afetados e, em consequência, a redução das coberturas vacinais se mantem por um longo período de tempo, o aparecimento de bolsões de suscetíveis são frequentes, favorecendo o ressurgimento das doenças infecciosas e dos óbitos relacionados a elas ${ }^{(16,26,35)}$. Isto pode ser constatado pelo aumento dos casos de poliomielite e difteria no Paquistão e Afeganistão ${ }^{(36)}$ e no aumento do número de casos de sarampo em todo o mundo ${ }^{(37)}$.

Ainda não se sabe quanto tempo essa pandemia durará ou como será o curso da propagação do vírus, entretanto, acredita-se que, por um período extenso, ela deve continuar a impor grandes cargas de morbidade e mortalidade, enquanto afeta gravemente as sociedades em todo o mundo. Dessa forma, os desafios trazidos pela COVID-19 e as estratégias adotadas para a sua mitigação não devem impedir que esforços sejam dispensados para a manutenção da vacinação de rotina e para a aceitabilidade das novas vacinas do SARS-CoV-2 ${ }^{(19,38)}$. O atual cenário é um lembrete da importância da vacinação como estratégia de prevenção e controle das doenças na população.

Desta forma, a OMS recomenda que todas as ações de vacinação de rotina sejam realizadas, respeitando as medidas e precauções de cada contexto social, e que quaisquer serviços de imunização interrompidos devem ser retomados e as vacinas ofertadas o mais rápido possível ${ }^{(39)}$. Ainda, se necessário, em uma fase posterior da pandemia, deve-se ofertar locais alternativos para vacinações de escolares ou da vacina contra influenza (19).

\section{O PAPEL DA ENFERMAGEM NO ENFRENTAMENTO DOS DESAFIOS AGRAVADOS PELA PANDEMIA NA VACINAÇÃO.}

No Brasil, mais precisamente no âmbito do SUS, o profissional de enfermagem é protagonista das ações de imunização, sendo responsável pelo manuseio, conservação e administração segura dos imunobiológicos, 
respondendo por todo o processo de trabalho na sala de vacina ${ }^{(40)}$. Assim, compete ao enfermeiro a responsabilidade técnica da gestão dos serviços de enfermagem em todos os estabelecimentos de saúde, que vão desde a coordenação, supervisão das ações desenvolvidas, até a educação permanente da equipe de vacinação, cabendo ao auxiliar e técnico de enfermagem a execução de atividades referentes a conservação e administração dos imunobiológicos ${ }^{(41-42)}$.

A confiança no sistema de saúde e o vínculo com os profissionais da saúde são considerados determinantes cruciais na adesão as recomendações do programa de imunizações ${ }^{(43)}$. Vários componentes de confiança são relevantes: confiança na vacina, confiança no fornecedor (laboratórios produtores) e confiança na política pública (sistema de saúde, governo e pesquisadores de saúde pública). Ainda, em situações de incerteza, em que o indivíduo deve decidir, com base na relação risco/benefício, pela aplicação ou não do imunizante, o vínculo e a confiança são condições indispensáveis para a tomada de decisão ${ }^{(8)}$.

Assim, é necessário que os programas de imunizações apresentem credibilidade diante da população, com uso de vacinas com comprovadas segurança e eficácia, rigoroso controle de qualidade da sua produção, adequada conservação e distribuição em todas as instâncias da cadeia de frio e o vínculo, boas práticas em vacinação e a confiança nos profissionais de saúde ${ }^{(44)}$. Nesse sentido, a Agência Nacional de Vigilância Sanitária (Anvisa) reforça que os serviços de vacinação tenham recursos humanos qualificados para desenvolver as atividades de vacinação durante toda a jornada de trabalho e que tenha um sistema de educação continuada, uma vez que a inserção de novas vacinas ou as mudanças nos esquemas de vacinas já incluídas no PNI são constantes ${ }^{(45)}$.

A confiança no profissional de saúde é primordial para o funcionamento efetivo do sistema de saúde. Essa relação tem papel importante na resposta à crise e a promoção do cumprimento das medidas em situações de emergência de saúde pública, uma vez que, são os profissionais de saúde, com destaque para a equipe de enfermagem, os encarregados em orientar os cuidados necessários para a proteção à saúde e a prevenção às doenças. Diante da recente crise, desencadeada pela pandemia da COVID-19, o vínculo com os profissionais de saúde tornou-se indispensável para o cuidado, o controle e a diminuição da disseminação do vírus.

Protagonista em todo o processo de combate a grave crise sanitária provocada pela COVID-19, o profissional da enfermagem se vê novamente no centro de uma importante ação de combate ao vírus, o processo de implementação/gestão da vacinação em massa contra o coronavírus. Envolvidos diretamente na operacionalização do processo de vacinação, a enfermagem desempenha um papel imprescindível na organização, administração da vacina e na orientação acerca dos benefícios e riscos do imunizante. Em cada esfera organizacional, suas atribuições sustentam e conduzem o planejamento das ações que visam atender a todos os grupos prioritários determinados pelo Ministério da Saúde.

\section{CONSIDERAÇÕES FINAIS.}

A sobrecarga ocasionada pela pandemia do COVID-19 impactou diretamente os sistemas de saúde em todo o mundo e gerou um aumento da carga de trabalho, inadequadas condições de serviço e falta de insumos e equipamentos de proteção individual, aumentando assim, o risco de adoecimento dos profissionais de saúde e comprometendo a atuação dos enfermeiros.

A pandemia afetou diretamente a logística e o funcionamento dos programas de saúde de rotina, como o de imunização, acarretando um declínio nas coberturas vacinais e favorecendo o reaparecimento das doenças. Por isso, é primordial atender com urgência às necessidades das unidades de saúde e retomar os serviços de saúde de rotina, respeitando as medidas preventivas do COVID-19, definindo uma estratégia integrada que possa envolver todas as entidades para salvaguardar os indivíduos e mitigar o impacto do surto (46).

A manutenção do funcionamento dos serviços de vacinação é fundamental para evitar a sobrecarrega ainda maior dos serviços de saúde por outras doenças imunopreveníveis ${ }^{(47)}$. O planejamento das ações deve ser 
baseado nas necessidades locais e no atual cenário epidemiológico da transmissão do novo coronavírus ${ }^{(48-49)}$. Ademais, várias estratégias devem ser recomendadas para melhorar a adesão e a captação dos indivíduos a serem imunizados, como a administração simultânea de múltiplas vacinas em cada visita e a utilização de intervalos mínimos entre as doses de vacinas em crianças com esquema vacinal em atrasado. Além de que, a vacinação extramuros, seja em locais estratégicos para evitar aglomerações ou no próprio domicílio, a ampliação do horário de funcionamento das unidades de saúde e a organização de local reservado, específico para o atendimento de grupos de maior risco com segurança, devem ser implementados ${ }^{(50)}$. Nesse contexto, os enfermeiros têm papel fundamental para o enfrentamento desse desafio imposto ao PNI pela pandemia.

\section{REFERÊNCIAS}

1. Domingues CMAS, Maranhão AGK, Teixeira AM, Fantinato FFS, Domingues RAS. The Brazilian National Immunization Program: 46 years of achievements and challenges. Cad Saude Publica. 2020;36(sup2):e00222919. https://doi. org/10.1590/0102-311X00222919

2. Barreto ML, Teixeira MG, Bastos FI, Ximenes RA, Barata RB, Rodrigues LC. Successes and failures in the control of infectious diseases in Brazil: Social and environmental context, policies, interventions, and research needs. Lancet [Internet]. 2011 [cited 2021 Mar 23];377(9780):1877-89. Available from: http://www.thelancet.com/article/S014067361160202X/fulltext

3. Waldman EA. Mesa-Redonda: Desigualdades sociais e cobertura vacinal: uso de inquéritos domiciliares. Rev Bras Epidemiol. 2008;11(suppl-1):129-32. Available from: https://doi.org/10.1590/S1415-790X2008000500013

4. Barata RB, Ribeiro MCSA, Moraes JC, Flannery B, Group behalf of the VCS 2007. Socioeconomic inequalities and vaccination coverage : results of an immunisation coverage survey in 27 Brazilian capitals, 2007 e 2008. J Epidemiol Community Health 2012;66:934-41. https://doi.org/10.1136/jech-2011-200341

5. Césare N, Mota TF, Lopes FFL, Lima ACM, Luzardo R, Quintanilha LF, et al. Longitudinal profiling of the vaccination coverage in Brazil reveals a recent change in the patterns hallmarked by differential reduction across regions. Int J Infect Dis. 2020;98:275-80. Available from: https://doi.org/10.1016/j.ijid.2020.06.092

6. Figueiredo A, Simas C, Karafillakis E, Paterson P, Larson HJ. Mapping global trends in vaccine confidence and investigating barriers to vaccine uptake: a large-scale retrospective temporal modelling study. Lancet. 2020;396(10255):898-908. https://doi.org/10.1016/S0140-6736(20)31558-0

7. Sato APS. Qual a importância da hesitação vacinal na queda das coberturas vacinais no Brasil? Rev Saude Publica. 2018;52(96):1-9. https://doi.org/10.11606/S1518-8787.2018052001199

8. MacDonald NE, Eskola J, Liang X, Chaudhuri M, Dube E, Gellin B, et al. Vaccine hesitancy: definition, scope and determinants. Vaccine. 2015;33(34):4161-4. Available from: https://doi.org/10.1016/j.vaccine.2015.04.036

9. Rasmussen SA, Smulian JC, Lednicky JA, Wen TS, Jamieson DJ. Coronavirus Disease 2019 (COVID-19) and pregnancy: what obstetricians need to know. Am J Obstetr Gynecol. 2020;222(5):415-26. https://doi.org/10.1016/j.ajog.2020.02.017

10. Sato APS. Pandemia e coberturas vacinais: desafios para o retorno às escolas. Rev Saude Publica. 2020;54(115):1-8. https:// doi.org/10.11606/s1518-8787.2020054003142

11. Smith KM, Machalaba CC, Seifman R, Feferholtz Y, Karesh WB. Infectious disease and economics: the case for considering multi-sectoral impacts. One Health. 2019;7(100080):1-6. https://doi.org/10.1016/j.onehlt.2018.100080

12. Morens DM, Fauci AS. Emerging Pandemic Diseases: how we got to COVID-19. Cell. 2020;182(5):1077-92. https://doi. org/10.1016/j.cell.2020.08.021

13. Ferguson NM, Laydon D, Nedjati-Gilani G, Imai N, Ainslie K, Baguelin M, et al. Report 9: impact of non-pharmaceutical interventions (NPIs) to reduce COVID19 mortality and healthcare demand. Imperial College COVID-19 Response Team. 2020. 20 p. https://doi.org/10.25561/77482

14. Prem K, Liu Y, Russell TW, Kucharski AJ, Eggo RM, Davies N, et al. The effect of control strategies to reduce social mixing on outcomes of the COVID-19 epidemic in Wuhan, China: a modelling study. Lancet Public Health. 2020;5(5):e261-70. https:// doi.org/10.1016/S2468-2667(20)30073-6

15. Moynihan R, Sanders S, Michaleff ZA, Scott AM, Clark J, To EJ, et al. Impact of COVID-19 pandemic on utilisation of healthcare services: A systematic review. BMJ Open. 2021;11(3):45343. https://doi.org/10.1136/bmjopen-2020-045343 
16. Nelson R. COVID-19 disrupts vaccine delivery. Lancet Infect Dis. 2020;20(5):546. https://doi.org/10.1016/ S1473-3099(20)30304-2

17. Horton R. Offline: COVID-19 and the NHS-"a national scandal". The Lancet. 2020;395:1022. https://doi.org/10.1016/ S0140-6736(20)30727-3

18. Zar HJ, Dawa J, Fischer GB, Castro-Rodriguez JA. Challenges of COVID-19 in children in low- and middle-income countries. Paediatr Respir Rev. 2020;35:70-4. https://doi.org/10.1016/j.prrv.2020.06.016

19. Dinleyici EC, Borrow R, Safadi MAP, van Damme P, Munoz FM. Vaccines and routine immunization strategies during the COVID-19 pandemic. Human Vaccin Immunother. 2020;17(2):400-7. https://doi.org/10.1080/21645515.2020.1804776

20. Schmid-Küpke NK, Matysiak-Klose D, Siedler A, Felgendreff L, Wieler L, Thaiss HM, et al. Cancelled routine vaccination appointments due to COVID-19 pandemic in Germany. Vaccine X. 2021;8:100094. https://doi.org/10.1016/j. jvacx.2021.100094

21. World Health Organization (WHO). Guiding principles for immunization activities during the COVID-19 pandemic [Internet]. 2020 [cited 2021 Mar 24]. Available from: https://www.who.int/emergencies/diseases/novel-coronavirus-2019/ technical-guidance

22. Pan American Health Organization (PAHO). The Immunization Program in the Context of the COVID-19 Pandemic [Internet]. 2020 [cited 2021 Mar 24]. Available from: https://www.paho.org/en/documents/ immunization-program-context-covid-19-pandemic-march-2020

23. World Health Organization. At least 80 million children under one at risk of diseases such as diphtheria, measles and polio as COVID-19 disrupts routine vaccination efforts, warn Gavi, WHO and UNICEF [Internet]. 2020 [cited 2021 Mar 24]. Available from: https://www.who.int/news/item/22-05-2020-at-least-80-million-children-under-one-at-risk-of-diseasessuch-as-diphtheria-measles-and-polio-as-covid-19-disrupts-routine-vaccination-efforts-warn-gavi-who-and-unicef

24. Sociedade Brasileira de Pediatria (SBP). Sociedade Brasileira de Imunizações (SBIm). Calendário vacinal da criança e a pandemia pelo coronavírus [Internet]. 2020 [cited 2021 Mar 24]. Available from: https://www.sbp.com.br/fileadmin/user_ upload/nt-sbpsbim-calendariodacrianca-pandemiacovid-200324.pdf

25. Weill A, Drouin J, Desplas D, Cuenot F, Dray-Spira R, Zureik M. Usage des médicaments de ville en France durant l'épidémie de la Covid-19 - point de situation jusqu'au 13 septembre 2020 [Internet]. Saint-Denis, França; 2020 [cited 2021 Mar 24 ]. Available from: https:/www.ameli.fr/sites/default/files/2020-10-05-dp-covid-19-usage-medicaments-13-septembre-2020.pdf

26. Moraga-Llop FA, Fernández-Prada M, Grande-Tejada AM, Martínez-Alcorta LI, Moreno-Pérez D, Pérez-Martín JJ. Recuperando las coberturas vacunales perdidas en la pandemia de COVID-19. Vacunas. 2020;21(2):129-35. https://doi. org/10.1016/j.vacun.2020.07.001

27. McDonald HI, Tessier E, White JM, Woodruff M, Knowles C, Bates C, et al. Early impact of the coronavirus disease (COVID-19) pandemic and physical distancing measures on routine childhood vaccinations in England, January to April 2020. Euro Surveill. 2020;25(19). https://doi.org/10.2807/1560-7917.ES.2020.25.19.2000848

28. Santoli JM, Lindley MC, DeSilva MB, Kharbanda EO, Daley MF, Galloway L, et al. Effects of the COVID-19 Pandemic on Routine Pediatric Vaccine Ordering and Administration[Internet]. United States: Centers for disease control and prevention; 2020;69(19):591-3. Available from: https://www.cdc.gov/mmwr/volumes/69/wr/mm6919e2.htm

29. Buonsenso D, Cinicola B, Kallon MN, lodice F. Child healthcare and immunizations in Sub-Saharan Africa during the COVID-19 Pandemic. Front Pediatr. 2020;8:517. https://doi.org/10.3389/fped.2020.00517

30. The Vaccine Alliance-GAVI. COVID-19 Situation Reports. COVID-19: Situation Report \#14 [Internet]. 2020 [cited 2021 Mar 29]. Available from: https://www.gavi.org/sites/default/files/covid/Gavi-COVID-19-Situation-Report-14-20200728-1.pdf

31. Mansour Z, Arab J, Said R, Rady A, Hamadeh R, Gerbaka B, et al. Impact of COVID-19 pandemic on the utilization of routine immunization services in Lebanon. PLoS One. 2021;16:1-11. https://doi.org/10.1371/journal.pone.0246951

32. Ministério da Saúde (BR). Secretaria de Vigilância em Saúde. Vigilância Epidemiológica do sarampo no Brasil - 2020: Semanas Epidemiológicas 1 a 32 [Internet]. 2020 [cited 2021 Mar 29]. Available from: https://antigo.saude.gov.br/images/ pdf/2020/August/31/Boletim-epidemiologico-SVS-34.pdf

33. Matos CCSA, Barbieri CLA, Couto MT. Covid-19 and its impact on immunization programs: reflections from Brazil. Rev Saude Publica. 2020;54(114):1-6. https://doi.org/10.11606/s1518-8787.2020054003042

34. Nunes L. Cobertura Vacinal do Brasil 2020 [Internet]. São Paulo; 2021 [cited 2021 Jun 7]. Available from: https://ieps.org.br/ wp-content/uploads/2021/05/Panorama_IEPS_01.pdf 
35. Stefanati A, d'Anchera E, De Motoli F, Savio M, Toffoletto MV, Gabutti G. Value of Immunizations during the COVID-19 Emergency. Int J Environ Res Public Health. 2021;18(2):778. https://doi.org/10.3390/ijerph18020778

36. Polio Global Eradication Initiative. This Week. Polio This Week as of 13 January 2020 [Internet]. 2020 [cited 2021 Mar 29 ]. Available from: https://polioeradication.org/polio-today/polio-now/this-week/

37. Roberts L. Why measles deaths are surging — and coronavirus could make it worse. Nature [Internet]. 2020 [cited 2021 Mar 29];580(7804):446-7. Available from: http://www.nature.com/articles/d41586-020-01011-6

38. Lazarus JV, Ratzan SC, Palayew A, Gostin LO, Larson HJ, Rabin K, et al. A global survey of potential acceptance of a COVID-19 vaccine. Nat Med. 2021;27(2):225-8. https://doi.org/10.1038/s41591-020-1124-9

39. World Health Organization (WHO). Routine Immunization Services during the COVID-19 Pandemic 23 . WHO guiding principles for immunization services during the COVID-19 pandemic 4 [Internet]. 2020 [cited 2021 Mar 29]. Available from: https://apps.who.int/iris/handle/10665/331561

40. Ministério da Saúde (BR). Secretaria de Vigilância em Saúde, Departamento de Vigilância das Doenças Transmissíveis. Manual de Normas e Procedimentos para Vacinação [Internet]. 2014 [cited 2021 Mar 30]. 178 p. Available from: https:// bvsms.saude.gov.br/bvs/publicacoes/manual_procedimentos_vacinacao.pdf

41. Conselho Federal de Enfermagem. Resolução COFEN no 0458/2014 - Normatiza as condições para Anotação de Responsabilidade Técnica pelo Serviço de Enfermagem e define as atribuições do Enfermeiro Responsável [Internet]. 2014[cited 2021 Mar 30]. 5 p. Available from: http://www.cofen.gov.br/resolucao-cofen-no-04582014_25656.html

42. Presidência da República (BR). L7498 - Dispõe sobre a regulamentação do exercício da enfermagem, e dá outras providências [Internet]. 1986[cited 2021 Mar 30]. 4p. Available from: http://www.planalto.gov.br/ccivil_03/leis/l7498.htm

43. Garcia ÉM, Palombo CNT, Waldman EA, Sato APS. Factors associated with the completeness of the vaccination schedule of children at 12 and 24 months of age in a Brazilian medium-size municipality. J Pediatr Nurs. 2021;S0882-5963(21)00070-1. https://doi.org/10.1016/j.pedn.2021.02.028

44. Kfouri RÁ, Petraglia TCMB, Lima EJF, Sato HK, Giamberarduni HI, Andrade SD, et al. Boas práticas em vacinação: evitando erros[Internet]. Rio de Janeiro. 2021 [cited 2021 Jun 06]. Available from: https://www.sbp.com.br/fileadmin/user_ upload/23045c-GPA-BoasPraticas_em_Vacinacao-_EvitandoErros.pdf

45. Agência Nacional de Vigilância Sanitária (Anvisa). Resolução - RDC No 197, de 26 de dezembro de 2017 [Internet]. Brasil; 2017[cited 2021 Mar 30]. 6p. Available from: https://www.in.gov.br/materia/-/asset_publisher/Kujrw0TZC2Mb/content/ id/1432311/do1-2017-12-28-resolucao-rdc-n-197-de-26-de-dezembro-de-2017-1432307

46. Lassi ZS, Naseem R, Salam RA, Siddiqui F, Das JK. The Impact of the COVID-19 Pandemic on Immunization Campaigns and Programs: a systematic review. Int J Environ Res Public Health. 2021;18(3):988. https://doi.org/10.3390/ijerph18030988

47. Bonanni P, Angelillo IF, Villani A, Biasci P, Scotti S, Russo R, et al. Maintain and increase vaccination coverage in children, adolescents, adults and elderly people: Let's avoid adding epidemics to the pandemic: Appeal from the Board of the Vaccination Calendar for Life in Italy: Maintain and increase coverage also by re-organizing vaccination services and reassuring the population. Vaccine. 2021;39(8):1187-9. https://doi.org/10.1016/j.vaccine.2020.10.024

48. Ministério da Saúde (BR). Orientações sobre o funcionamento dos serviços de vacinação do Sistema Único de Saúde no contexto da pandemia de COVID-19. Ofício no 173/2020. CGPNI/DEIDT/SVS/MS. Abril, 2020 [Internet]. Brasília-DF; 2020[cited 2021 Mar 30]. Available from: https://sbim.org.br/images/files/notas-tecnicas/sei-ms--0014289729---oficio1732020-cgpni-deidt-svs-ms.pdf

49. Olusanya OA, Bednarczyk RA, Davis RL, Shaban-Nejad A. Addressing Parental Vaccine Hesitancy and Other Barriers to Childhood/Adolescent Vaccination Uptake During the Coronavirus (COVID-19) Pandemic. Front Immunol [Internet]. 2021 [cited 2021 Apr 27];12:1. Available from: https://doi.org/10.3389/fimmu.2021.663074

50. Ministério da Saúde (BR). Secretaria de Vigilância em Saúde, Departamento de Imunização e Doenças Transmissíveis, Programa Nacional de Imunizações. Estratégia de recuperação do esquema de vacinação atrasado de crianças menores de 5 anos de idade. Brasilia-DF; 2020. 